\title{
Sixth Immunotherapy of Cancer conference (ITOC): advances and perspectives $-a$ meeting report
}

\author{
Catarina Pinto, ${ }^{1}$ Michael Bergmann, ${ }^{2}$ Daria Briukhovetska, ${ }^{3}$ Volkmar Nuessler, ${ }^{3,4}$ \\ Mario Sznol, ${ }^{5}$ Michael von Bergwelt-Baildon, ${ }^{6,7}$ Sebastian Kobold ${ }^{3,7}$
}

To cite: Pinto C, Bergmann M, Briukhovetska D, et al. Sixth Immunotherapy of Cancer conference (ITOC): advances and perspectives-a meeting report. Journal for ImmunoTherapy of Cancer 2020;8: $\mathrm{e} 000268$. doi:10.1136/jitc-2019-000268

- Additional material is published online only. To view please visit the journal online (http://dx.doi.org/10.1136/jitc2019-000268).

$\mathrm{CP}, \mathrm{MB}$ and $\mathrm{DB}$ contributed equally.

Accepted 19 December 2019

Check for updates

(C) Author(s) (or their employer(s)) 2020. Re-use permitted under CC BY-NC. No commercial re-use. See rights and permissions. Published by BMJ.

For numbered affiliations see end of article.

\section{Correspondence to}

Professor Sebastian Kobold; sebastian.kobold@med.unimuenchen.de

\section{ABSTRACT}

Immunotherapy has moved to the forefront of cancer treatment, illustrated by the accelerating pace of novel therapy approvals. In this complex environment, scientists rely on cutting edge conferences to stay informed.

The Immunotherapy of Cancer (ITOC) conference was established jointly with the Society of the Immunotherapy of Cancer to bring the European researchers together. In its sixth edition, the ITOC conference has recently been held in Vienna, Austria.

\section{PREFACE}

The past years have seen an explosion in the number of indications approved for immunotherapeutic modalities and new clinical trials. ${ }^{12}$ The present report highlights the novel aspects presented during the plenary sessions (figure 1). This year Immunotherapy of Cancer (ITOC) dedicated a lifetime award to Alberto Mantovani to recognize his input to the understanding of the mechanisms of innate immunity and inflammation in cancer.

\section{MICROENVIRONMENT AND METABOLISM}

Emanuel Donnadieu observed that $\mathrm{T}$ cells actively excluded from the tumor accumulate in the peritumoral region. Ex vivo microscopy revealed that $\mathrm{CD} 206^{+}$macrophages reduce $\mathrm{T}$ cell motility, and dense matrix fibers prevent cell infiltration. However, ICAM1 expression on the tumor cells promoted CAR T cell infiltration.

Eduard Batlle observed that cancer-associated fibroblast cell programme is associated with prognosis in colorectal cancer (CRC) and driven by transforming growth factor-beta (TGF- $\beta$ ) signaling. Model of mouse CRC using organoids with Lgr5, APC, KRAS, Tgfbr2, and Trp53 mutations demonstrated therapeutic effects of TGF- $\beta$ neutralization.

Bo Huang demonstrated that PCK1-mediated gluconeogenesis is essential for memory $\mathrm{T}$ cell formation and maintenance through the increase of reduced glutathione and decrease of reactive oxygen species (ROS). Moreover, cancer cells may mechanically resist cell lysis by myosin-based contraction.

While studying $\mathrm{T}$ cell metabolism, Pedro Romero revealed that memory $\mathrm{T}$ cells are essential for melanoma control. VLA1 ${ }^{+}$tumorinfiltrating lymphocytes (TILs) controlled the tumor outgrowth and were found to coexpress CD69 and CD103, indicating a tissueresident phenotype. Additionally, $\mathrm{TCF} 1^{+} \mathrm{CD}^{+}$ memory $\mathrm{T}$ cell frequency in patient samples correlated with a good prognosis.

Lisa Derosa identified that Akkermansia muciniphila and Bacteroides salyersiae present in the stool of non-small-cell lung carcinoma (NSCLC) and renal cell carcinoma patients are associated with favorable clinical responses to treatment. In murine models, these species reversed resistance to PD-1/ CTLA-4 blockade after fecal transplantation from non-responder patients.

\section{EMERGING CONCEPTS AND NEW AGENTS}

Using single-cell RNA profiles, Ana Anderson found that checkpoint blockade therapyinduced transcriptional changes in Tim-3 PD-1 ${ }^{-}$CD $8^{+}$TILs. Memory precursor subset of those TILs identified as CD62LSlamf$7^{\text {hi }} \mathrm{CX} 3 \mathrm{CR} 1^{-}$the population is controlled by TCF1, and its loss limits the response to checkpoint blockade.

Paolo Ascierto showed that elevated expression of CD73 and its activity correlates with a low response rate to nivolumab and ipilimumab treatment. This concept was previously found valid for melanoma patients and is currently tested in prostate cancer.

Claudia Lengerkeshowed that chemotherapyresistant LSCs lack NKG2D ligands that are necessary for NKG2D-mediated natural killer (NK) cell killing. PARP1 suppresses the expression of those ligands, and its inhibitors could be used as therapeutic agents to control leukemogenesis in acute myeloid leukemia. 
- Matrix fibers and T cell exclusion

- Stromal cells and TGF- $\beta$

- T memory cell metabolism

- Microbiome and immunotherapy
- Transcriptional changes in TILs

- CD73 and checkpoint inhibition

- NK cell receptor ligands

- Deglycosilation

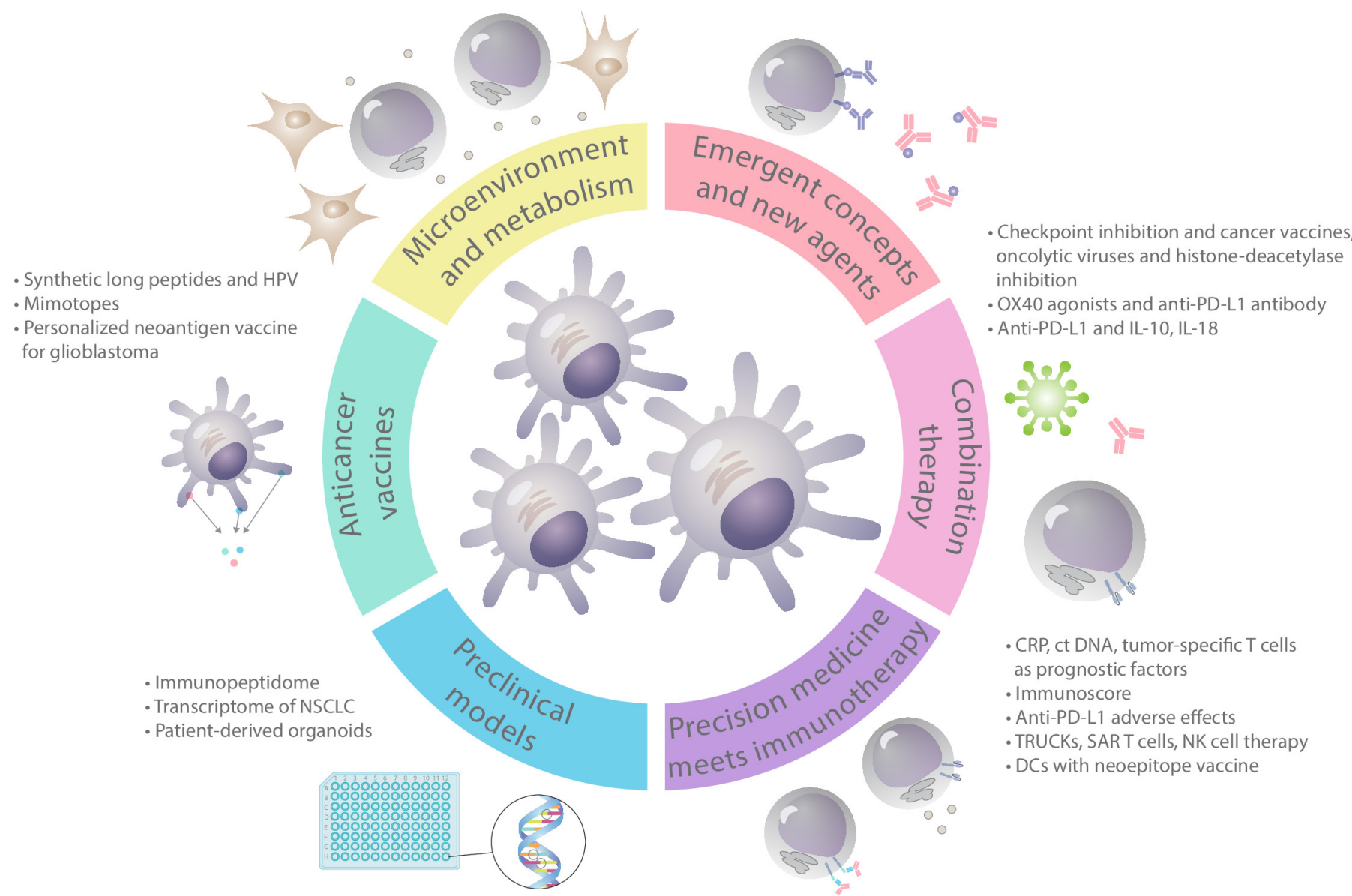

Figure 1 Summary of the sessions presented at the sixth Immunotherapy of Cancer conference. CRP, C-reactive protein; DC, dendritic cells; IL, interleukin; NK, natural killer; NSCLC, non-small-cell lung carcinoma; PD-L1, programmed death-ligand 1 ; RCC, renal cell carcinoma; SAR, synthetic agonist receptor; TGF- $\beta$, transforming growth factor-beta; TIL, tumor-infiltrating lymphocyte; TRUCKS, T cells redirected for antigen-unrestricted cytokine-initiated killing.

NKG2A, a receptor for HLA-E that suppresses NK cell function, is an attractive target for immunotherapy. Stephanie Cornen combined NKG2A blockade with cetuximab to target microsatellite stable colon cancer and head and neck squamous cell carcinoma.

Finally, glycosylation is shaping the interactions of cells with their microenvironment. Siglec-9 is a lectin that is upregulated in different tumor entities, which affect macrophage polarization. Heinz Läubli showed that tumor-specific antibodies coupled to sialidases are effective at delaying tumor growth.

\section{COMBINATION THERAPY}

Sjoerd van der Burg proposed to combine checkpoint inhibition with vaccines directed against human papillomavirus in oropharyngeal cancers. Moreover, the combination of carboplatin and paclitaxel depleted myeloid cells, which effectively induced strong and sustained $\mathrm{T}$ cell responses.
Samir Khleif highlighted that the combination of OX40 agonists and programmed death-ligand 1 (PD-L1) antibody therapy mutually inhibited each other. However, MEK inhibition decreases $T$ cell exhaustion, which can potentially be used to enhance the therapeutic efficacy of OX40 agonists.

Efficacy of IL-18 therapy is hampered by IL18-BP, a soluble decoy receptor. Aaron Ring engineered a decoyresistant interleukin 18 (IL-18) variant that showed efficacy as a single agent and in combination with anti-PD1 antibodies in several tumor models eliciting memory like $\mathrm{TCF}^{+}{ }^{+} \mathrm{CD} 8^{+} \mathrm{T}$ cells.

IL-18 is also induced by PEGylated IL-10 (Pegliodecakin), which has been shown to enhance immune activation when combined with anti-PD-L1 therapy. Aung Naing presented data from a phase I trial supporting the observed effect, but side effects raise safety concerns.

Alan Melcher demonstrated that systemic administration of oncolytic reovirus induces immunogenic tumor cell death. It avoids neutralizing antibodies utilizing protective 
cell carriage by monocytes, enhances their APC function, and improves the efficacy of checkpoint inhibitor blockade.

Alfred Budillon presented early phase clinical trials suggesting that epigenetic modifiers can potentiate the response to checkpoint inhibitors. Histone-deacetylase inhibitor therapy appears to be associated with an increase in immunogenic cell death, a decrease in Tregs, and downregulation of c-myc.

\section{PRECLINICAL MODELS}

Krijn Dijkstra cocultured peripheral-blood-derived T cells with patient-derived organoids to enrich tumor-reactive $\mathrm{T}$ cells and analyze tumor-specific $\mathrm{T}$ cell responses to epithelial cancers. Such T cells showed no cross-reactivity with healthy tissue organoids as assessed by CD137 expression.

Using single-cell RNA sequencing, Bernard Thienpont presented the transcriptome clustering of cells that shape the microenvironment of NSCLC. He identified stromal cells as the major contributor that are further divided into 52 clusters. The comprehensive data array is also available for endothelial, epithelial, and immune cells in online ( gbiomed.kuleuven.be/scRNAseq-NSCLC).

Angela Krackhardt used mass spectrometry to analyze the immunopeptidome from melanoma patients and identify neoepitopes. She showed that zirconium-89 labeled neoantigen-specific TCR transgenic $\mathrm{T}$ cells could be tracked by PET imaging in mice and humans.

\section{Precision medicine meets immunotherapy}

Jeffrey Weber has identified C-reactive protein (CRP) and IL- 6 as negative prognostic factors shared among different malignancies. CRP has been shown to suppress $\mathrm{T}$ cell activation.

Francois-Clément Bidard proposed to assess tumor mutational burden analyzing ctDNA in plasma as compared with tissue biopsies with higher sensitivity and lower failure rate.

Han $\mathrm{Si}$ analyzed ctDNA in metastatic NSCLC patients treated with anti-PD-L1 and anti-CTLA4 therapies. He used the Guardant Health OMNI platform, a highly sensitive 500-gene sequencing test, to stratify patients according to their TMB score to predict checkpoint inhibition outcome.

High T-cell infiltration is a favorable prognostic factor in breast cancers, including triple-negative breast cancer (TNBC). Barbara Seliger combined PD-L1-blockade with chemotherapy, which promoted immune infiltration into TNBC.

Immune checkpoint inhibitors can induce lifethreatening toxicity due to myocarditis. Lei Zheng used animal models to study and predict dilated cardiomyopathy due to PD-1, PD-L1/PD-L2 deletion, or anti-PD-L1 antibody treatment.

Jerome Galon utilized high-throughput methods to discover critical parameters of the immune response that together compose the immune contexture of tumors: nature, functional orientation, density, and localization of immune cell populations, which are combined as
Immunoscore. It was used to identify CRC patients with a high risk of recurrence.

Alessandra Nardin performed analysis of PBMCs from patients treated with atezolizumab (PD-L1-blockade) in the POPLAR trial and revealed that the majority of unique neoantigen $\mathrm{T}$ cells were present in the patients that responded to treatment. Hence, tumor-specific $\mathrm{T}$ cells could be used as a prognostic marker of therapy outcome.

\section{CELL THERAPY}

Lisa Butterfield presented the current advancements in the development of next-generation cell therapies, including affinity-enhanced T cells (NY-ESO-1 ${ }^{\mathrm{c} 259}$ TCR in metastatic synovial sarcoma, H3.3K27M-specific TCR in diffuse midline gliomas), combinatorial antigen recognition CAR T cells, dendritic cell-based personalized multipeptide neoepitope vaccines.

Hinrich Abken presented T cells redirected for antigenunrestricted cytokine-initiated killing that secret CARinducible IL-12 or IL-18 and target CEA antigen in pancreatic cancer. They indirectly promote the killing of $\mathrm{CEA}^{-}$cancer cells. CAR T cells with the inducible secretion of IL-7 and an artificial IL-7R/IL-2R $\beta$ can be rendered resistant to TGF- $\beta$-mediated immunosuppression.

Sebastian Kobold presented $\mathrm{T}$ cells bearing synthetic agonist receptor (SAR) as a new modular cellular therapy platform to overcome the issues of antigen restriction. Cross-linking bispecific antibody conditionally activates SAR T cells in the presence of tumor-specific antigens, which enables a large variety of antigens to be targeted by a single genetically modified $\mathrm{T}$ cell.

Ulrike Koehl gave insights into the current developments of NK cell-based therapies. Currently, multiple trials employ NK donor lymphocyte infusion (NK-DLI), autologous and allogeneic CAR NK cells (anti-CD19 and CD123 against leukemic cells), redirected "CAR" NK-92 cell line and additional IL-2 stimulation.

\section{ANTI-CANCER VACCINES}

Cornelis Melief proposed to use synthetic long peptides that stimulate $\mathrm{CD} 4^{+}$and $\mathrm{CD} 8^{+}$positive $\mathrm{T}$ cells to target human papilloma virus (HPV)-associated cancers and particularly in head and neck cancers, in combination with nivolumab and ipilimumab.

Ursula Wiedermann-Schmidt demonstrated that Her-2/ neu specific mimotopes are efficient and safe for vaccination against breast cancer.

David Reardon presented results of a phase I study of a personalized neoantigen cancer vaccine for glioblastoma that is designed to overcome the effects of tumor evolution. However, this concept could still not overcome the immunosuppression generated by the tumor.

\section{SUMMARY}

While combinatorial treatments currently lead the pace of clinical development, novel strategies are still emerging. 
Along with a growing understanding of the complexity of cancer and the immune system, we are looking forward to the new approaches in the field. The upcoming ITOC7 conference to be held in Munich from 2 April to 4 April 2020.

\section{Author affiliations}

${ }^{1}$ Human Cancer Immunology, Boehringer Ingelheim RCV GmbH \& Co KG, Vienna, Austria

${ }^{2}$ Department of Surgery, Medical University of Vienna, Vienna, Austria

${ }^{3}$ Center of Integrated Protein Science Munich (CIPS-M) and Division of Clinical Pharmacology, Klinikum der Ludwig-Maximilians-Universitat Munchen, Munich, Germany

${ }^{4}$ Tumor Center Munich, Munich, Germany

${ }^{5}$ Yale Cancer Center, Yale University, New Haven, Connecticut, USA

${ }^{6}$ Department of Medicine III, Klinikum der Ludwig-Maximilians-Universitat Munchen, Munich, Germany

${ }^{7}$ German Consortium for Translational Cancer Research (DKTK), Munich, Germany

Acknowledgements We thank Nicholas Tokarew, $\mathrm{PhD}$, for support in language editing.

Contributors All authors have contributed to writing the manuscript and have approved the final version.

Funding SK was supported by grants from the international doctoral programme "i-Target: Immunotargeting of Cancer" funded by the Elite Network of Bavaria, the
Melanoma Research Alliance (grant number 409510), the Marie-Sklodowska-Curie "Training Network for the Immunotherapy of Cancer (IMMUTRAIN)" funded by the H2020 program of the European Union, the Else Kröner-Fresenius-Stiftung, the German Cancer Aid, the Ernst-Jung-Stiftung, by LMU Munich's Institutional Strategy LMUexcellent within the framework of the German Excellence Initiative, by the Bundesministerium für Bildung und Forschung, by the European Research Council Starting Grant (grant number 756017), by the DFG, the Hector foundation and the José-Carreras Cancer Foundation.

Competing interests SK has received speakers honorarium from GSK and Novartis. SK has served on the scientific advisory board of Celyad SA and TCR2. SK's research is supported in parts by TCR2.

Patient consent for publication Not required.

Provenance and peer review Not commissioned; externally peer reviewed.

Open access This is an open access article distributed in accordance with the Creative Commons Attribution Non Commercial (CC BY-NC 4.0) license, which permits others to distribute, remix, adapt, build upon this work non-commercially, and license their derivative works on different terms, provided the original work is properly cited, appropriate credit is given, any changes made indicated, and the use is non-commercial. See http://creativecommons.org/licenses/by-nc/4.0/.

\section{REFERENCES}

1 Kobold S, Krackhardt A, Schlösser H, et al. [Immuno-Oncology: A Brief Overview]. Dtsch Med Wochenschr 2018;143:1006-13.

2 Tang J, Shalabi A, Hubbard-Lucey VM. Comprehensive analysis of the clinical immuno-oncology landscape. Ann Oncol 2018;29:84-91. 